\title{
What Motor Truck Would Suit Me Best?
}

\section{A Composite List of American Motor Trucks and Delivery Wagons Arranged According to Carrying Capacity}

\author{
Compiled by Theodore M. R. von Kéler
}

70 arrange commercial motor vehicles according 1 to price is almost impossible, as the great majority of both heavy and light cars are furnished with bodies suited to the special needs of the purchaser. This list, therefore, has been based upon the carrying capacity, and the prices in most instances apply to the chassis, fitted with driver's seat.

Bodies in a great variety of forms are furnished by the manufacturers at prices ranging from $\$ 100$ upward.

The majority of truck manufacturers are willing to sell to small merchants on easy payments, and to supervise the installation of motor service by means of their own service departments.

As in the list of pleasure cars published last week, the many small companies of purely local importance have been omitted, and only the leading models have been listed. Besides the factories named herein there are nearly one hundred companies which have been formed within the last six months, and whose prospective output will not fall short of 5,000 trucks during the coming year. As yet their product has not reached a stage which would warrant its listing in a national periodical.
Capacity 500 to 1,000 Pounds. Anderson Elec. Car Co., Detrolt, Mich. (\$2,345) Anderson Elec. Car Co., Detroit, Mich. (\$2,345)
Bessemer Motor Truck Co., Grove City, Pa.
$(\$ 1,250)$
Bowling Green Motor Car Co., Bowling Green, Bowling
$0 . \quad(\$ 1,200)$ Chase Motor Truck Co, Syracuse, N. Y. (\$500) Commerce Motor Car Co., Detroit, Mich. $(\$ 750)$

Dart Mfg. Co., Waterloo, Iowa. (\$750) Durant-Dort Carrage Co., Filnt, Mich. (\$750) General Vehicle Co., Long Island City, N. Y.

Hupp Motor Car Co., Detroit, Mich. (\$950) McIntyre, w. H., Co., Auburn, Ind $(\$ 1,500)$

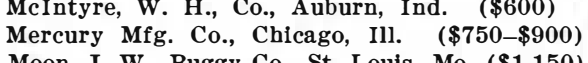
Moon, J. W., Buggy Co., St. Louis, Mo. $(\$ 1,150)$
Studebaker Corp., South Bend, Ind. $(\$ 1,500)$ Sullivan Motor Car Co., Rochester, N. Y. (\$925)

U. S. Motor Co., Detroit, Mich. $(\$ 1,400)$ Ward Motor

Waverly Co., Indianapolis, Ind. $(\$ 1,800)$

Capacity from 1,001 to 2,000 Pounds. Adams Bros. Co., Findlay, Ohio. (\$2,100) $(\$ 2,000)$

Anderson Elec. Car Co., Detroit, Mich. $(\$ 2,870)$ Atterbury Motor Car Co., Buffalo, N. (\$1,50)

Available Truck Co., Chicago, Ill. $(\$ 900-\$ 1,350)$ Avery Co., Peoria, III. $(\$ 2,000)$ (1) $(\$ 1,800)$

wling Green

Q. Brown Commercial Car Co., Peru, Ind. $(\$ 1,650)$ Buick Motor Co., Flint, Mich. (\$1,000-\$1,400) Chase Motor

Chicago Pneumatic Tool Co., Chicago, III. roce Auto Co., Asbury Park, N. J. $(\$ 1,850)$ Dart Mfg. Co., Waterloo, Iowa. (\$1,100) Durant-Dort Carriage Co., Flint, Mich. $(\$ 1,375)$
General Vehicle Co., Long Island City, N. Y $(\$ 1,710)$

Gramm Motor Truck Co., Lima, O. (\$1,750)

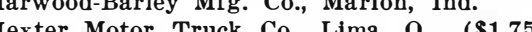
Hupp Motor Car Co., Detroit, Mich. (\$750) deal Auto Co., Fort Wayne, Ind. $\$ \$ 1,750$ $\$ 2,000)$

International Motor Co., New York city.

$(\$ 2,500)$
Kelly-Springfield Motor Truck Co., Springfield, Kissell Motor Car Co., Hartford, Wis. $(\$ 1,500)$ Knickerbocker Motor Truck Mfg. Co., New York city. $(\$ 2,000)$

Koebler, H. J., Co., New York city. (\$750) Krebs Commercial Car Co., Clyde, O. $(\$ 1,375)$ Lauth-,Juergens Motor Car Co., Fremont, Ohi $(\$ 2,100)$

(\$1,650-\$1,675)

(\$1,650-\$1,675) Cor National Motor Truck $(\$ 1,225)$

Nyberg Automobile Works, Anderson, Ind. $(\$ 1,300)$

Oakland Motor Car Co., Detroit, Mich. (\$1,500) Piggins Motor Truck Co., Racine, Wis. (\$1,750 Poyer, D. F., Co., Menomimee, $\$ 1,500$

Reo Motor Truck Co., Lansing, Mich. (\$750) Rowe Motor Mfg. Co., Coatesville, Pa. (\$1,700$\$ 2,100)$
nosky Motor Truck Co., Sandusky, Obio

Sanford Motor Truck Co., Syracuse, N. Y. elden Truck Sales Co., Rochester, N. Y. Co., Wabash, Ind. $(\$ 1,350$

$(\$ 1,475)$
$(\$ 1,650)$ Studebaker Corp., South Bend, Ind. $(\$ 2,000)$
Sullivan Motor Car Co., Rochester, N. Y. Sullivan Motor Car Co., Rochester, N. Y.
$(\$ 1,050)$

$(\$ 1,850)$ \begin{abstract}
Universal Motor Truck Co., Milwaukee, Wis.
(\$1,950)
Velie Motor Vehicle Co., Moline, Ill. $(\$ 2,000)$
Ward Motor Vehicle Co., New York city.
$(\$ 2,000)$
Waverly Co., Indianapolis, Ind. $(\$ 2,150)$ White Co., Cleveland, O. $\quad(\$ 2,100)$
Wilcox Motor Car Co., Minneapolis, Minn $\$ 2,300$ )
ilcox
\end{abstract}

Capacity from 2,001 to 3,000 Pounds. Adams Bros. Co., Findlay, Obio. $(\$ 2,300)$ Adams Bros. Co., Findlay, Obio. $(\$ 2,300)$
Alden-Sampson Mfg. Co., Detroit, $(\$ 2,500)$
Electric Car Co., Detroit, Mich. $(\$ 3,132.50)$

terbury Motor Car Co., Buffalo, N. Y. $(\$ 2,300)$

Auto Car Co., Ardmore, Pa. (\$2,150)
Bessemer Motor Truck Co., Grove City, Pa. $(\$ 2,100)$

Blair Mfg. Co., Newark, O. (\$3,000)
Bowling Green Motor Co., Bowling Green, o.

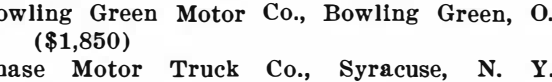
$(\$ 1,750)$

Croce Auto Co., Asbury Park, N. J. ( $\$ 2,500)$ Dart Mfg. Co., Waterloo, Iowa. (\$1,800)

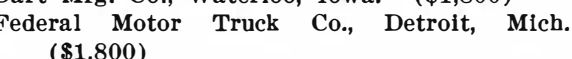

$(\$ 1,800)$
Four-Wheel Drive Co., Clintonville, wis.
$(\$ 3,600)$ $(\$ 3,600)$
eneral Motors Co., Detroit, Mich. $(\$ 2,075)$ Grand Rapids Motor Truck Co., Grand Raplds Mich. $(\$ 2,200-\$ 2,500)$
Co., Marion, Ind $(\$ 2,000)$

Ideal Auto Co., Fort Wayne, Ind. $(\$ 2,250)$

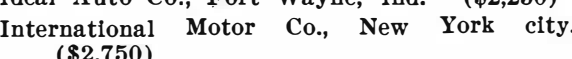
Juno Motor Truck Co., Juneau, Wis. $(\$ 2,200)$

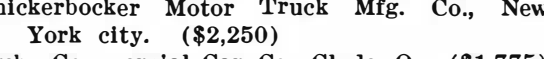
Krebs Commercial Car Co., Clyde, o. (\$1,775)
Mais Motor Truck Co., Indianapolis, Ind. $(\$ 2,750-\$ 2,800)$

McIntyre, W. H., Co., Auburn, Ind. $(\$ 2,300)$ Moon, J. W., Buggy Co., St. Louis, Mo. (\$1,900) Piggins Motor Truck Co., Racine, Wis. $(\$ 2,250)$
Reo Motor Truck Co., Lansing, Mich. $(\$ 1,800)$ Rowe Motor Mfg. Co., Coatesville, Pa. $(\$ 2,850)$ Sanford Motor Truck Co., Syracuse, N. Y.
$(\$ 1.910)$

Service Motor Co., Wabash, Ind. $(\$ 1,675)$ Studebaker Corp., South Bend, Ind. $(\$ 2,950)$
Transit Motor Truck Co., Loulsville, Ky. nsit Motor Truck Co., Louisville, Ky.
$(\$ 2,300)$ (\$2,

$(\$ 2,750)$

Walter Motor, Detroit, Mich. (\$2.200) $(\$ 2,800)$

Whte Co., Cleveland, O. $\$ 3,000$

Wilcox Motor Car Co., Minneapolis, Min

Capacity from 3,001 to 4,000 Pounds. Adams Bros. Co., Findlay, Ohio. $(\$ 2,500)$ (\$2,800)

\section{$(\$ 2,800)$
erican Lo}

$(\$ 2,950)$

$(\$ 2,650)$

ery Co., Peoria, Ill. (\$2,700)

Chase Motor Truck Co., Syracuse, N. Y.
$\begin{aligned} & (\$ 2,200) \\ & \text { Croce Auto Co., Asbury Park, N. J. }(\$ 2,850)\end{aligned}$ Garford Co., Elyria, O. $(\$ 2,800)$ General Vehicle Co., Long Island City, N. Y $(\$ 2,090)$

Gramm-Bernstein Co., Lima, O. (\$2,750) Gramm Motor Truck Co., Lima, O. (\$2,600) rwood-Bar

$(\$ 2,500)$

Hexter Motor Truck Co., Lima, O. $(\$ 2,600)$ Ideal Auto Company, Ft. Wayne, Ind. (\$2,500) $(\$ 3,000)$

Juno Motor Truck Co., Juneau, Wis. $(\$ 2,800)$
Kissell Motor Car Co., Hartford, Wis. $(\$ 2,750)$ nox Auto Car Co., Hartford, Wis. $(\$ 2,750)$ Knox Auto. Co., Springfield, Mass. (\$3,000)
Lauth-Juergens Motor Car Co., rremont, $(\$ 2,800)$ $(\$ 2,800)$ (Truck C

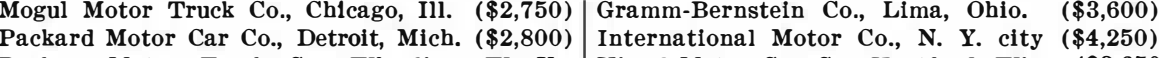

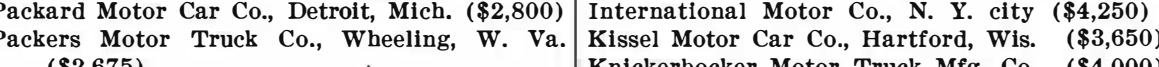
(\$2,675)
Piggins Motor Truck Co., Racine, Wis. $(\$ 2,750)$ Reo Motor Truck Co., Lansing, Mich. (\$1,950) Rowe Motor Mfg. Co., Coatesville, Pa. $(\$ 3,100)$
Smith, A. O., Co.. Milwaukee, Wis. $(\$ 3,000)$ Smith, A. O., Co.. Milwaukee, Wis.
Speedwell Motor Car Co., Dayton, O. Sternberg Motor Truck Co., Milwaukee, Wis. $(\$ 2,800)$

Studebaker Corp., South Bend, Ind. (\$3,300) urban Truck Co., $1420 \mathrm{C}$
delphia, Pa. $(\$ 2,750)$

ransit Motor Truck Co Louisville, $\mathrm{K}_{\mathrm{y}}$ $(\$ 2,850)$

versal Motor Truck Co., Milwaukee, Wis. elie Motor Vehicle Co., Moline, Ill. $(\$ 2,850)$ alter Motor Truck Co., New York city. $(\$ 3,000)$

ard Motor Vehicle Co., New York city. $(\$ 2,900)$
averly Co., Indianapolis, Ind. $(\$ 3,000)$

Capacity from 4,001 to 6,000 Pounds. Alden-Sampson Motor Car Co., Detroit, Mich. $(\$ 3,400)$
$(\$ 3,550)$ $(\$ 3,350)$
Co., Peoria, Ill. $(\$ 3,200)$

Blair Mfg. Co., Newark, $0 . \quad(\$ 3,250)$

(

(\$3,600) Company, Asbury Park, N. J. (\$2,800) Truck Co., Detroit, Mich. $(\$ 2,800)$ $(\$ 4,000)$

Garford Co., Elyria, O. (\$3,500)

ramm Motor Truck Co., Lima, O. $\quad(\$ 3,500)$ (\$3,200)
(\$) Hexter Motor Truck Co., Lima, o. (\$3,500) International
$(\$ 3,750)$

Juno Motor Truck Co., Juneau, Wis. $(\$ 3,400)$ Kelly-Springfield Motor Truck Co., Springfield O. $\quad(\$ 3,400)$

Kartford, Wis. $(\$ 3,350$ nickerbocker Motor Truck Mfg. Co., New
York city. $(\$ 3,500)$

$(\$ 3,700)$
$(\$ 3,450)$

$(\$ 3,450)$
$(\$ 30)$

$(\$ 3,450)$
Motor
$(\$ 3,400)$

$(\$ 3,400)$
McIntyre, W. H., Co., Auburn, Ind. (\$3,200) Packard Motor Car Co., Detroit, Mich. $(\$ 3,400)$
Peerless Motor Car Co., Cleveland, O. $\$ 3,700)$ Peerless Motor Car Co., Cleveland, O. $(\$ 3,700)$ Rowe Motor Mfg. Co., Coatesville, Pa. $(\$ 3,400)$
Standard Motor Truck Co., Detroit, Mich. $(\$ 2.750)$ Service Motor Car Co., Wabash, Ind. (\$2,475)
Sternberg Motor Truck Co., Milwaukee, Wis. $(\$ 3,400)$

\section{versal Motor Tran}

. S. Motor Co., Detroit, Mich. $(\$ 3,400)$

Velie Motor Vehicle Co., Moline, Ill. $\mathbf{( \$ 3 , 3 5 0 )}$
Victor Motor Truck Co, Buffalo, Wictor Motor Truck Co., Buffalo, N. Y. (\$2,750)
Walter Motor Truck Co., New York city. $(\$ 3,400)$
white Co., Cleveland, O. $\quad(\$ 3,700)$

ilcox Motor Car Co., Minneapolis, Min $(\$ 3,250)$
$(\$ 4$, Chu

$(\$ 4,750)$

Capacity from 6,001 to 8,000 Pounds. Iden-Sampson Motor Car Co., Detroit, Mic $(\$ 4,250)$
$(\$ 3,650)$

$\underset{\$ 3,650)}{\text { erican Locomotive Co., Schenectady, N. Y. }}$ Anderson Elec. Car Co., Detroit, Mich.
Blair Mfg. Co., Newark, O. $(\$ 3,750)$

Couple-Gear Freight wheel Co., Grand Rapids Mich. $(\$ 4,400)$
Garford Co., Elyria, O. $(\$ 3,850)$

Garford Co., Elyria, O. $(\$ 3,850)$
General Motors Co., Detroit, Mich. $(\$ 3,500$ $\$ 3,750$ )

$(\$ 2,620)$
$(25)$

$\begin{array}{ll}\text { Knickerbocker Motor Truck Mfg. Co. } & (\$ 4,000) \\ \text { Mogul Motor Truck Co., Chicago, Ill. } & (\$ 3,800)\end{array}$ Mogul Motor Truck Co., Chicago, Ill. $(\$ 3,800)$
Packers Motor Truck Co., Wheeling, w. Va.

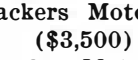

Peerless Motor Car Co., Cleveland, O. $(\$ 4,000)$ mith, A. O., Co., Milwaukee, Wis. $(\$ 3,750)$ peedwell Motor Car Co., Dayton, O. $(\$ 3,750)$

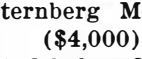

Studebaker Corp., South Bend, Ind. $(\$ 3,500)$ Transit Motor Truck Co., Louisville, Ky. $(\$ 3,500-\$ 3,750)$
Walter Motor Truck Co., N. Y. city. $(\$ 3,750)$ rd Motor Vehicle Co., New York city. $(\$ 3,400)$

Waverly Co., Indianapolis, Ind. (\$3,500)
Wyckoff, Church \& Partridge, New York city. $(\$ 5,250)$

Capacity from 8,001 to 10,000 Pounds. Aires Motor Truck Co., N. Y. city. $(\$ 5,250)$ Alden-Sampson Motor Car Co., Detroit, Mich. $(\$ 4,750)$

erican Locomotive Co., Schenectady, N. Y $(\$ 4,750)$

erbury Motor Car Co., Buffalo, N. Y $(\$ 4.250)$
Co., Peoria, Ill. $(\$ 4,500)$

Couple-Gear Freight Wheel Co., Grand Rapids,

Croce Auto Co., Asbury Park, N. J. $\quad(\$ 4,500)$ Garford Co., Elyria, O. $\quad(\$ 4,500)$ General Vehicle Co., Long Island City, N. Y.

Gramm Motor Truck Co., Lima, O. $(\$ 4,500)$ 


\section{New-York Life Insurance Co.}

346 Broadway, New York

\section{SIXTY-EIGHTH YEAR OF BUSINESS}

\section{To the Policy-holders:}

Your Directors assume that, when you think of your contract with this Company, you never question the Company's soundness, but that you are deeply interested in its progress, and in the efficiency and economy of its management.

We submit, therefore. the following summary from the transactions of the year:

During 1912 the Company received in premiums - _ - _ - . - - \$85,941,784.05

In Interest, Rents, etc. - - - - - - - - - - - - - - 33 33,301,582.53

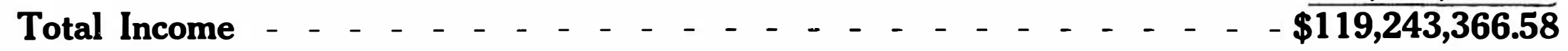

\section{INVESTMENTS MADE DURING YEAR}

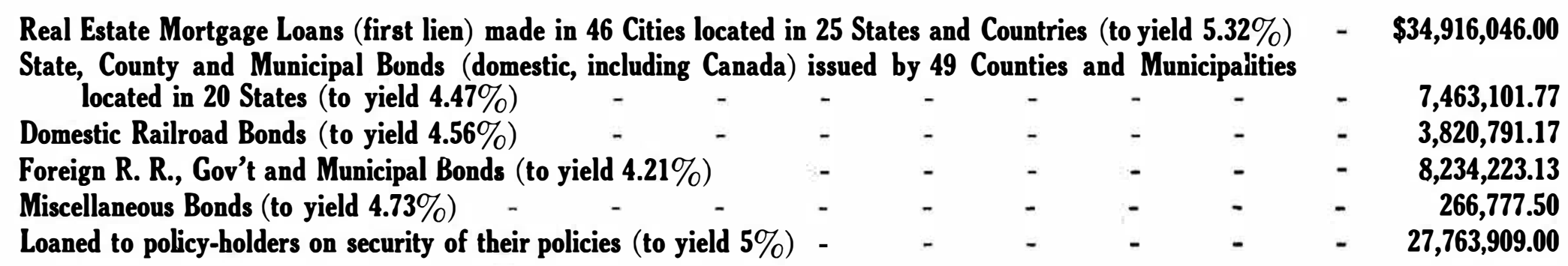

\section{DURING 1912 THE INSURED OR THEIR BENEFICIARIES RECEIVED FOR}

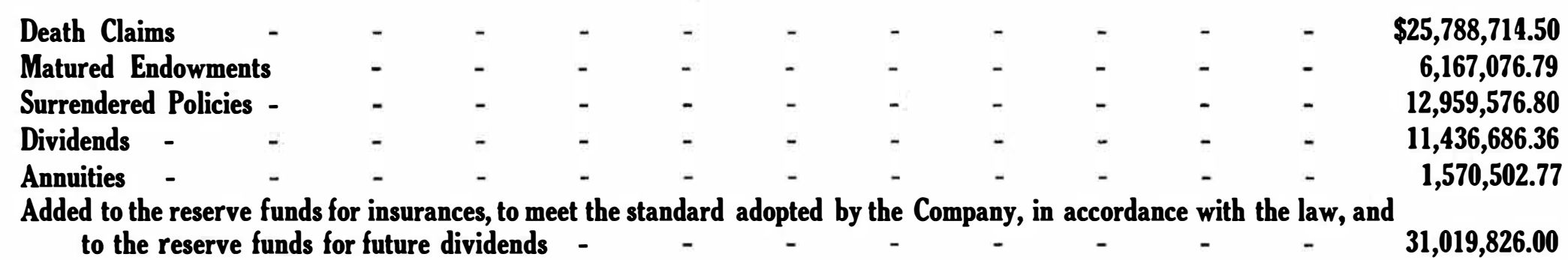

The increase in the earning power of the Company's assets during the last seven years is equal to 0.29\%. Translated into dollars this means an increase in earning power, over 1905, of more than TWO MILLION DOLLARS.

The increased earning power developed in 1912 is notable. It is represented by $9 / 100$ of $1 \%$, and, if maintained; will increase the income of the Company in 1913 by comparison with what it would have been had the earning power remained as at the close of 1911, by the sum of - $\quad-\quad \$ 647,000$

Of the amount which the law allowed us to spend in 1912 for new business, we actually spent ... $91 \%$

Of the amount which the law allowed us to spend for all purposes, we spent approximately .... $63 \%$

Of the amount of new business which the law allowed us to issue in 1912 , we issued .......

\section{NINETEEN-TWELVE WAS A GOOD YEAR}

IF YOU DESIRE FURTHER DETAILS, WRITE THE HOME OFFICE, 346 BROADWAY, NEW YORK.

January 8, 1913.

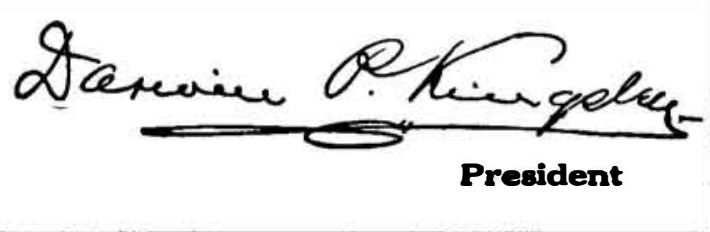

